\title{
Lidil
}

Revue de linguistique et de didactique des langues

Sémantique des noms et adjectifs d'émotion

\section{Synonymie et incompatibilité des noms d'émotions}

Gerda Haßler

\section{OpenEdition}

Journals

Édition électronique

URL : http://journals.openedition.org/lidil/94

DOI : $10.4000 /$ lidil.94

ISSN : $1960-6052$

Éditeur

UGA Éditions/Université Grenoble Alpes

Édition imprimée

Date de publication : 1 décembre 2005

ISBN : 2-914176-13-9

ISSN : $1146-6480$

\section{Référence électronique}

Gerda Haßler, «Synonymie et incompatibilité des noms d'émotions », Lidil [En ligne], 32 | 2005, mis en

ligne le 18 juillet 2007, consulté le 19 avril 2019. URL : http://journals.openedition.org/lidil/94 ; DOI

10.4000/lidil.94

Ce document a été généré automatiquement le 19 avril 2019

(C) Lidil 


\title{
Synonymie et incompatibilité des noms d'émotions
}

\author{
Gerda Haßler
}

1 La synonymie est considérée comme une relation de similarité sémantique entre des mots, moins stricte qu'une identité sémantique totale (Geerarts, 1994: 4455) et impliquant aussi des cas d'identité partielle. On peut définir la synonymie par rapport aux lexèmes et par rapport aux occurrences de mots dans un contexte. La première approche vise à une comparaison de la gamme entière des significations des mots en question et utilise comme critère la substituabilité de deux mots dans tous les contextes possibles. Deux lexèmes sont des synonymes partiels s'ils ne sont interchangeables que dans quelques contextes. Ils peuvent présenter des traits sémantiques incompatibles avec des contextes dans lesquels l'autre synonyme est parfaitement possible.

2 Pour les mots qui désignent des mouvements psychiques, on peut constater qu'ils fonctionnent comme synonymes dans certains contextes, mais qu'ils ont aussi des emplois spécifiques. Nous analyserons les mots étonnement, émerveillement, surprise et stupéfaction dans le but de déterminer leur synonymie à partir d'un examen de leur profil combinatoire en contexte.

Méthode et corpus utilisés

3 Le terme de profil combinatoire a été introduit par Peter Blumenthal qui le définit comme « la structure schématique du voisinage syntaxique et sémantique d'un mot telle qu'elle se manifeste dans un vaste corpus » (Blumenthal, 2002 : 116). Nous ne recourrons pas aux méthodes mathématiques appliquées par Blumenthal, Diversy et Mielebacher (2005), mais nous essaierons de déterminer les différences entre les mots en question à partir de leur cooccurrence dans le corpus. Nous partons de l'hypothèse que l'étude minutieuse des cooccurrences de deux ou plusieurs synonymes dans un contexte peut aider à approfondir la caractérisation des valeurs d'un mot face aux mots sémantiquement voisins. Le corpus utilisé pour cette analyse sera FRANTEXT que nous utilisons pour une étude panchronique sur tous les genres, mais qui exclut les textes antérieurs à 1700 . Quand deux ou plusieurs mots désignant la même notion apparaissent ensemble dans un contexte, la question à poser est de savoir s'il s'agit de variation stylistique ou de 
différenciation sémantique. Après l'étude panchronique des cooccurrences, nous procéderons à une analyse sur un corpus plus restreint par le genre et le temps de la production. GLOSSANET est un corpus électronique qui rassemble, entre autres, une vingtaine de journaux de langue française. Comme les données ne s'archivent pas, nous avons étudié les mots en question dans les éditions de novembre 2004 jusqu'à février 2005, ce qui a donné un corpus de 4020 occurrences des quatre mots. Il est problématique, bien sûr, de s'appuyer sur deux corpus différents, mais l'objectif de l'étude basée sur GLOSSANET n'est que la vérification des résultats et des traits spécifiques dans un corpus actuel.

4 En discutant la signification des synonymes partiels, Philip Edmonds et Graeme Hirst constatent des différences de plusieurs niveaux entre leurs qualités sémantiques: « Usually words that are close in meaning are near-synonyms (or plesionyms) - almost synonyms, but not quite; very similar, but not identical, in meaning: not fully intersubstitutable, but instead varying in their shades of denotation, connotation, implicature, emphasis or register.» (Edmonds \& Hirst 2002: 107). Le phénomène de synonymie totale étant marginal à la suite de l'élimination de synonymes totaux d'une langue, nous utiliserons le terme synonyme pour des synonymes partiels. Les dimensions dans lesquelles la signification des synonymes peut varier sont les suivantes.

5 a) Sur le plan conceptuel, on peut constater une variation des dénotations des mots. C'està-dire que les différences entre les synonymes ne concernent pas seulement quelques traits de la présentation d'un objet, mais le concept même.

b) Les mots peuvent être différents dans la manière de présenter un concept. Ils peuvent l'introduire par suggestion ou implication ou exprimer des différences stylistiques. Nous appellerons ce niveau sous-conceptuel plan sémantico-stylistique.

c) Il faut y ajouter des différences sur le plan sémantico-syntaxique. Les mots en question peuvent remplir des fonctions différentes dans une phrase et, souvent, les différences dépendent du contexte.

Suivant Wittgenstein (1984: 563) qui veut que l'usage enseigne la signification des mots, le principe d'analyse sémantique sera l'étude de la signification dans les contextes. Cela exclut, par exemple, l'élimination d'un sens d'un mot polysémique. S'il y a co-occurrence avec un voisin sémantique, le contexte sera pris en compte.

Les dimensions de la variation. Interchangeabilité ou incompatibilité

9 Avant de commencer l'étude des cooccurrences des mots étonnement, émerveillement, surprise et stupéfaction, il faut savoir si ces mots sont interchangeables ou si le contexte peut bloquer l'emploi de certains d'entre eux. Les mots étudiés désignent un 'mouvement psychique'qui représente le noyau notionnel de leurs significations. Le mouvement psychique est pris comme point de repère onomasiologique pour la comparaison sémantique des noms d'émotions étudiés. Il correspond à un changement de la situation mentale d'une ou de plusieurs personnes, changement qui peut être considéré par rapport à ces personnes mêmes (experiencers) ou par rapport aux causes de ce mouvement qui a lieu dans l'espace et dans le temps et auquel correspond une certaine durée. On peut y ajouter la dimension évaluative du sentiment exprimé (affect positif/négatif), la variation de sa force et, peut-être, l'insistance sur son caractère résultatif ou progressif (dimension « aspectuelle»).

10 Dans son livre Semantics, Culture and Cognition (1992), Anna Wierzbicka a exprimé son scepticisme à l'égard d'un ensemble d'émotions humaines telles que 'intérêt', 'joie', 
'surprise', 'tristesse' qui seraient universelles et bien définies. Elle a élaboré un métalangage qui utilise un nombre restreint de notions élémentaires qui composent les concepts de la pensée humaine (Wierzbicka, 1996). L'universalité doit s'exprimer en termes d'un métalangage sémantique qui ne dépende pas des conditions lexicales d'une langue particulière. Malgré son désir d'objectivité, Wierzbicka trouve les notions élémentaires de la description de façon intuitive. Nous ne prétendons pas utiliser des notions vraiment élémentaires, mais nous partons du cadre sémantique qui est ouvert par la notion générale de 'mouvement psychique'. Avec les notions élémentaires 'cause du mouvement' et 'objet de l'emportement', un premier cadre semble trouvé auquel on peut ajouter les dimensions de l'appréciation', de la 'force' et de la 'durée' qui, de leur côté, sont caractérisées par un degré plus ou moins élevé.

11 Si l'on veut décrire les noms qui expriment des émotions en français, il faut se rendre compte que la répartition du contenu conceptuel entre des mots est arbitraire et ne dépend pas entièrement de causes extralinguistiques. La dénomination de certaines émotions et sentiments dépend du jeu de délimitation des valeurs entre les mots en question qui n'est jamais terminé et qui efface les limites entre synonymie et incompatibilité.

12 L'analyse conceptuelle et les tests de substitution ont donné les éléments suivants qui varient dans les synonymes, sur la base commune ('mouvement psychique') :

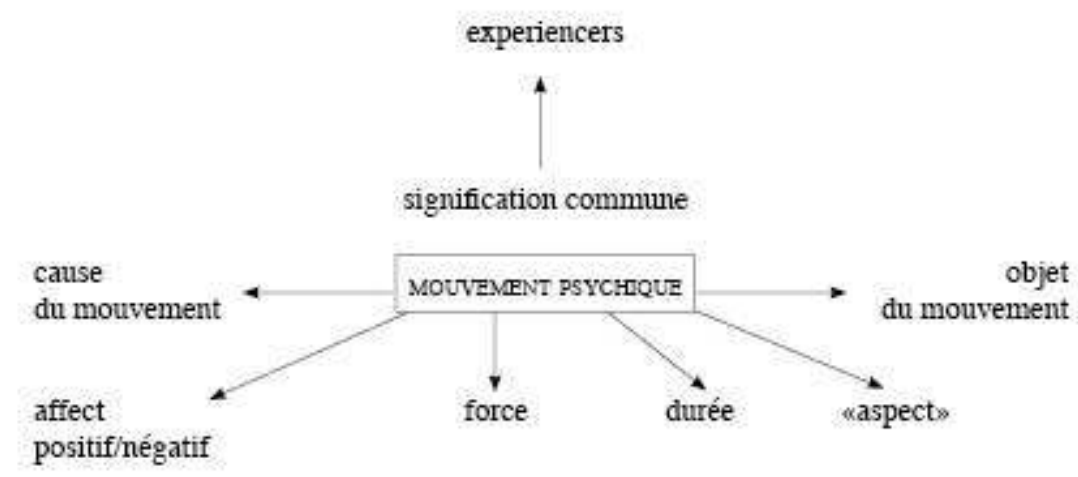

Dans le cas de surprise, la polysémie relie le mouvement et la cause de ce mouvement. Le Dictionnaire de l'Académie donne dans toutes ses éditions comme première signification 'action par laquelle on surprend', mais il mentionne qu'il signifie aussi 'étonnement', 'trouble'. Dans l'édition de 1932-1935, il donne une explication plus élaborée de cette signification : «Il signifie aussi Étonnement, état de l'esprit qui est frappé par quelque chose d'inattendu». À partir d'une dénomination de 'la chose qui surprend; ce qui provoque l'étonnement', surprise est devenu par métonymie un mot qui renvoie à l'état et à l'objet de cet état. Le Trésor de la langue française présente étonnement comme synonyme dans cette acception.

14 En mettant au centre de l'étude les dénominations d'un 'mouvement psychique', nous avons exclu les nombreuses occurrences dans lesquelles surprise désigne le fait qui provoque ce mouvement. Néanmoins, la polysémie est parfois présente dans un même contexte et doit être prise en compte. 
15 La relation à la cause du mouvement psychique dans étonnement et émerveillement est présente par la structure morphologique, ce qui s'explique par l'héritage de la valence des verbes, obligatoire pour le sujet, mais non pour l'objet direct :

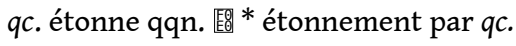

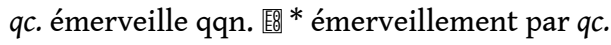
substitution dans un contexte qui permet aussi l'interprétation négative. Dans l'exemple (3) la possibilité de substitution d'émerveillement, qui désigne un sentiment positif, se limite à admiration. La substitution par étonnement, surprise ou stupéfaction contredirait le sens manifestement positif d'émerveillement :

(3) S'il est un miracle de cette civilisation qui a su compter jusqu'à deux mille ans, il réside dans notre émerveillement devant la naissance de chaque humain, quel qu'il soit.

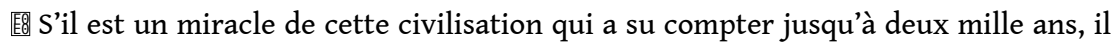
réside dans notre admiration devant la naissance de chaque humain, quel qu'il soit. 渇 S'il est un miracle de cette civilisation qui a su compter jusqu'à deux mille ans, il réside dans notre étonnement (surprise ou stupéfaction) devant la naissance de chaque humain, quel qu'il soit.

L'évaluation positive peut donc être vérifiée, grâce au test de substitution, qui montre qu'il s'agit d'un trait distinctif d'émerveillement, l'opposant à étonnement, surprise et stupéfaction. sémantique qui peut donner des résultats, comme ici pour identifier les dimensions aspectuelles ou évaluatives. Mais ce procédé de substitution n'est jamais exempt de jugements subjectifs. L'appui de l'analyse sémantique sur une étude d'un corpus peut mener à plus d'objectivité et rediriger vers l'usage réel.

Co-occurrences de termes psychiques

Si deux termes psychiques sont utilisés dans un énoncé, on peut supposer que l'énonciateur fait une différence entre les deux, même s'ils sont synonymes, ou qu'il les emploie pour des raisons stylistiques, c'est-à-dire pour éviter une répétition. On trouve des constructions qui ne relèvent pas de la dépendance syntaxique et dans lesquelles les 
synonymes se trouvent simplement coordonnés. Dans cette position, ils peuvent exprimer des rapports d'incompatibilité, de renforcement réciproque, d'addition et d'alternative (Blumenthal 2002: 122). L'étude des cooccurrences des mots étonnement, émerveillement, surprise et stupéfaction à partir des cooccurrences trouvées dans FRANTEXT a donné les résultats suivants.

Variation stylistique et synonymie « absolue »

Il y a quelques cas de simple variation stylistique, mais ceux-ci ne représentent pas la majorité des emplois de deux des termes en question. La variation stylistique s'emploie dans le cas de deux experiencers différents auxquels le même sentiment psychique est attribué :

(4) Ma mere aprit votre histoire avec étonnement, elle en fit part sur le champ à mon pere, dont la surprise fut encore plus grande ; (P246/Mouhy, Charles de/La Paysanne parvenue ou les Mémoires de Mme la Marquise de L. V./1735, 247)

L'identification du sentiment éprouvé par des personnes différentes peut être explicite et permettre ainsi l'usage synonymique absolu :

(5) Tonton se mit à souffler dedans, d'abord tout doucement, et, malgré l'étonnement que provoqua en moi cette musique inattendue, j'observai que Pélo et le grand-père avaient relevé la tête, tous les deux, presque en même temps, saisis de la même surprise (S368/Guilloux, Louis/Le Pain des rêves/1942, 131)

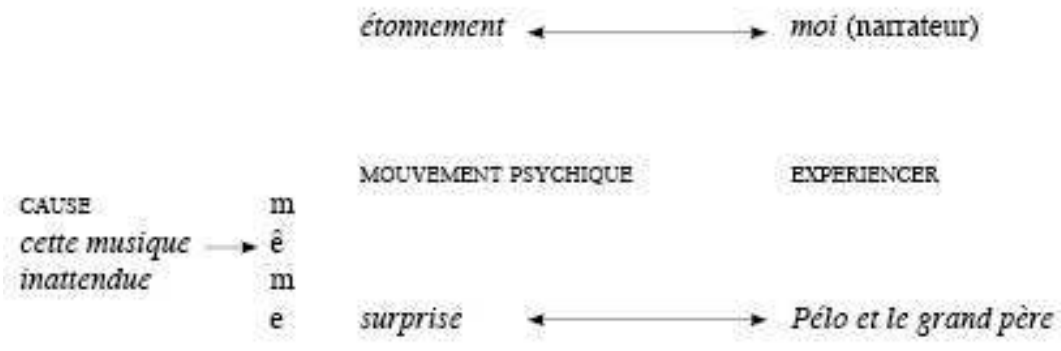

On peut classifier, de la même manière, comme variation stylistique l'usage de termes différents employés dans un texte littéraire soit par le narrateur, soit par un personnage :

(6) Après un moment de silence, pendant lequel la belle patricienne et l'ouvrière misérable s'étaient mutuellement examinées avec une surprise croissante, Adrienne dit à la Mayeux : - La cause de notre étonnement à toutes deux est, je crois, facile à deviner ; (S406/Sue Eugène/Le Juif errant/1845, 405)

Des synonymes dans des énumérations

La différenciation sémantique des synonymes partiels commence déjà dans leur usage dans une énumération qui, dans la langue écrite et littéraire ne peut pas être considérée comme simple paraphrase par un auteur à la recherche d'un mot. Des exemples comme (7) à (10) présupposent une différence de signification entre surprise et étonnement qui n'est pas explicitée :

(7) l'individu sera frappé de surprise, d'étonnement, d'admiration, de crainte, de terreur ou d'effroi. (M538/Maine de Biran/De l'influence de l'habitude sur la faculté de penser/1803, 90)

(8) D'où la surprise, l'étonnement, la grâce, tout ce que je t'ai déjà raconté. (S246/

Ormesson, Jean d'/ La Douane de mer/1993, 334) 
(9) Il n'y avait d'ailleurs, dans ma sensation, ni surprise ni étonnement. (R829/Gautier, Théophile/Mademoiselle de Maupin/1836, 118)

(10) Lucien, qui vit pour la première fois le luxe parisien fonctionnant, marchait ainsi de surprise en surprise, et cachait son étonnement en homme d'esprit, de coeur et de style qu'il était, selon le mot de Blondet. (M754/Balzac Honoré de/Les Illusions perdues/1843, 401) quelques emplois définitoires de termes psychiques qui concrétisent la distance sémantique d'une manière très individuelle, mais qui reflètent les caractéristiques de l'usage général. Dans (15) la différence de degré est située sur la dimension de la durée, ce qui se fait par l'attribution de l'adjectif soudain à surprise et pour la définition d'étonnement par l'hypéronyme surprise avec l'épithète longue.

(15) La surprise est un ébranlement soudain à la vue d'une nouveauté. L'étonnement une surprise longue et accablante; l'admiration, une surprise pleine de respect. La plupart de ces sentiments ne sont pas trop composés, et n'affectent pas aussi durablement (S014 Vauvenargues/ Introduction à la connaissance de l'esprit humain/1747)

Dans (16), trois termes psychiques se trouvent mis en relation, en établissant un lien d'égalité entre admiration et étonnement, mais en ajoutant l'adjectif accidentel. Le trait 
positif d"agréable'est ajouté comme épithète de surprise qui, dans ce cas, joue sur la polysémie du sentiment lui-même et de la cause du mouvement psychique.

(16) L'admiration n'est, ce me semble, qu'un étonnement accidentel de notre intelligence à l'occasion d'une surprise agréable. (M462/Bernardin de Saint-Pierre/

Harmonies de la nature : t. 2/1814, 266)

Usage anaphorique

Le sens plus large de surprise est confirmé par la reprise anaphorique d'autres termes psychiques :

(17) Un éveil subit accentua brusquement ma surprise, et c'est ce qui me permit de me souvenir si bien de ce rêve. Mon étonnement, à mon réveil, mon émerveillement, fut, en y repensant, d'avoir si bien su favoriser cette surprise. (K625/Gide, André/ Journal : 1889-1939/1939, 896)

Contiguïté sémantique

Aux différenciations de degré se joignent les descriptions de passages entre les sentiments qui font voir la contiguité sémantique des termes. Dans (18) surprise et étonnement se trouvent nettement opposés, surprise mis en opposition à tranquillité, et étonnement à tristesse, ces oppositions caractérisent en même temps une possibilité de passage.

(18) Depuis que le camion de la blanchisserie était passé le prendre chez sa grandmère, deux jours plus tôt, pour ces premières vacances de sa vie, il allait de surprise en étonnement. (S248/Poirot-Delpech, Bertrand/ L'Été 36/1984, 64)

(19) [...] son visage passe successivement de la joie folle à la joie modérée, de cette joie à la tranquillité, de la tranquillité à la surprise, de la surprise à l'étonnement, de l' étonnement à la tristesse, de la tristesse à l'abattement, de l'abattement à l'effroi, de l'effroi à l'horreur, de l'horreur au désespoir. (N363/Diderot Denis/Paradoxe sur le comédien/1784, 328)

Le passage d'un sentiment à l'autre renvoie à un concept de mouvement psychique qui se trouve différencié par des mots qui ont des particularités sémantiques. Ce sont des dénominations d'états contigus qui permettent le passage de l'un à l'autre, mais la direction de ce passage est déterminée : c'est toujours l'état plus concret ou plus intense qui est la fin de ce passage (surprise $\diamond$ stupéfaction) :

(20) [...] à mesure que je chantais, la petite pie s'éloignait de moi d'un air de surprise qui devint bientôt de la stupéfaction, puis qui passa à un sentiment d'effroi accompagné d'un profond ennui. (M896/Musset, Alfred de/Histoire d'un merle blanc/1842,57)

(21) La surprise de Paganel devint de la stupéfaction. (M256/Verne, Jules/Les Enfants du Capitaine Grant $/ 1868,130$ )

Différences de cause et d'experiencer

Dans des textes qui discutent des problèmes de l'entendement humain, des différences s'établissent aussi à partir de la cause du mouvement psychique (22) ou de son porteur ( experiencer) (23) :

(22) Si vous y regardez de près, vous trouverez que les objets qui causent de l' étonnement ou de l'admiration sans faire plaisir ne sont pas beaux, et que ceux qui font plaisir sans causer de la surprise ou de l'admiration, ne le sont pas davantage. (N552/Diderot, Denis/Salon de 1767/1768, 142)

(23) Siècle d'étonnement pour les ames vulgaires, de surprise et d'effroi pour les tyrans, d'affranchissement pour un grand peuple, et d'espérance pour toute la terre! (M683/Volney/Les Ruines ou Méditations sur les révolutions des empires/1791, 110) 
L'exemple (23) montre déjà que le mot surprise se prête aussi à la désignation de sentiments négatifs. Comme étonnement, il se trouve assez souvent dans le voisinage sémantique de termes explicitement négatifs (antipathie, horreur) (24), (25) ainsi qu'avec des termes positifs (26), ce qui prouve son caractère neutre par rapport à l'évaluation du sentiment :

(24) Godeau quelque part dans un groupe, surprise, étonnement, antipathie des autres à lui, de lui aux autres. (K786/ Jouhandeau, Marcel/ Monsieur Godeau intime/ 1926, 107)

(25) Le mieux c'était de jouer la surprise, l'étonnement... l'horreur. (S984/Céline, Louis-Ferdinand/Mort à crédit/1936, 1022)

(26) Ou, plutôt que la surprise, l'émerveillement, le ravissement, l'enchantement. (S322/

Romilly, Jacqueline de/Les (Eufs de Pâques/1993, 46)

Ce caractère neutre de surprise découle, peut-être, de sa signification primaire: dénomination de la cause, le mot devient par métonymie signe d'un sentiment positif ou négatif. Dans (27), surprise est la cause des sentiments opposés que sont antipathie et émerveillement.

(27) La princesse de Parme était Courvoisier par l'incapacité d'innover en matière sociale, mais, à la différence des Courvoisier, la surprise que lui causait perpétuellement la duchesse De Guermantes engendrait non comme chez eux l' antipathie, mais l'émerveillement. (K443/Proust, Marcel/À la recherche du temps perdu. 9. Du côté de Guermantes 2/1921, 469)

La différenciation entre la cause de l'étonnement et l'objet de l'admiration est explicite dans quelques emplois :

(28) Partagés entre l'admiration pourtant de sainteté et l'étonnement que causeront à leur droit sens tant de bizarreries, nos hérö̈ques et judicieux compatriotes essaieront en vain d'éteindre la discussion. (R644/Renan, Ernest/Marc Aurèle et la fin du monde antique : le règne de Marc-Aurèle $(161-180) / 1881,142)$

41 Surprise est le mot avec l'intension minimale 'mouvement psychique provoqué par quelque chose d'inattendu ou d'extraordinaire'qui peut être considéré comme hyperonyme d'étonnement, d'émerveillement et de stupéfaction. L'étonnement exprime un plus haut degré de force, encore renforcé dans la stupéfaction, qui est un étonnement extrême privant de toute réaction. Émerveillement représente un cas d'intersection avec un autre champ lexical, renvoyant à un sentiment complexe mêlé de plaisir et d'approbation (admiration, adoration).

Termes psychiques dans les journaux de langue française

Après avoir discuté les co-occurrences dans FRANTEXT, il est intéressant de vérifier les résultats dans le corpus des journaux GLOSSANET. L'analyse ne se limitera pas aux cooccurrences, mais prendra en compte toutes les apparitions des mots étudiés.

Dans la majorité des énoncés avec surprise, ce mot désigne la cause du mouvement psychique. Il entre dans quelques syntagmes fréquents et stéréotypés, comme par exemple belle surprise :

(29) «Je ne m'attendais vraiment pas à être primé. C'est une belle surprise!", s'exclame Cédric, quelques minutes avant de recevoir sa récompense des mains du ministre au Centre de formation des apprentis de Meaux. (Le Parisien, 2005/01/13).

Les cas d'occurrences de surprise comme dénomination de mouvements psychiques sont rares et souvent suivis de citations :

(30) Les économistes n'ont pas caché leur surprise en découvrant ces chiffres, mais ils tiennent néanmoins à nuancer la situation. «Au premier semestre, les prix ont 
bondi de 11,9\%, mais au second semestre, ils n'ont progressé que de 2,8\% ». (Le Figaro, 2005-01-10) mentionnées pour des textes littéraires et jouant sur la position contiguë à émerveillement

(31) Quant à Monique Souveine, elle a l'art et la manière de présenter les artistes : " Étonnement, plaisir, surprise... c'est un plaisir réel de rencontrer une artiste qui parle de ses tableaux par le biais des poètes, qui les commente avec des mots etc. » ( Le Progrès de Lyon, 2005-01-10)

(32) Emerveillement devant la complexité de la vie et la multiplicité des points de vue. Les interrogations sur le sens de la vie, le monde, la liberté, la mort, la beauté, ... sont partagées par tous. Cet étonnement n'a pas d'âge : les enfants, tout petits, interrogent le monde qui les entoure : " et pourquoi ceci ?", « et comment ça se fait que?». En stimulant cet étonnement, en retrouvant la simplicité de moment, les choses paraissent-elles plus complexes qu'au premier abord? Poser une question revient à accepter que les choses n'aillent pas de soi. Pour philosopher, on rentre dans un état de doute, d'étonnement, d'émerveillement. Émerveillement devant la complexité de la vie et la multiplicité des points de vue. Les interrogations sur le sens de la vie, le monde, la liberté, la mort, la beauté,... (La Libre Belgique, 2005-01-11)

Cependant, une certaine péjoration du sens d'étonnement est aussi à constater : le mot exprime déjà par lui seul un sentiment négatif (33), (34), tout en gardant un sens positif dans d'autres contextes (35) et exprimant aussi un mouvement psychique fort, mais sans connotation positive ou négative (36). Il peut donc être considéré en soi comme neutre :

(33) Les voleurs avaient cette fois là mis à sac les bureaux et tenté d'ouvrir le coffre contenant l'argent pour le portage des repas à domicile, mais sans succès. Parmi le personnel, hier, c'était l'étonnement en se demandant quand aura lieu le prochain passage des vandales... (Le Journal de l'ile de la Réunion, 2005-01-12)

(34) Il a fait part de son étonnement au ministre de l'Économie, des Finances et de l'Industrie, Hervé Gaymard, sur la modification, par son ministère, de l'âge légal des bénéficiaires de la demi-part du quotient familial [...] (Le Bien Public, 2005-01-17)

(35) Car il s'agit bien toujours de croissance et de nouveaux investissements. Pour continuer d'attirer les foules et rester un royaume magique offrant un étonnement permanent à ses visiteurs nouveaux et anciens (les taux de retours chez Disney sont toujours très élevés), les parcs se doivent d'évoluer, d'ajouter sans cesse de nouvelles attractions, [...] (L'Est républicain, 2005-01-14)

(36) Chaque diapositive ne passait à l'écran qu'accompagné d'un commentaire de Paul Baudras qui, dans le noir et sans texte préparé, faisait revivre spontanément chaque image. Alors, étonnement et émotion parcouraient la salle, où une cinquantaine de personnes s'appliquait soudain à découvrir ou reconnaître tel bâtiment ou tel parent ou ami. Avec 30 ans de moins! (Le Journal de Saône-et-Loire, 2005-02-02)

Dans sa signification moderne, le mot étonnement couvre une large gamme de mouvements psychiques, positifs ou négatifs, forts ou faibles, et il s'utilise aussi comme euphémisme pour désigner un agacement. Cette signification domine dans le corpus des journaux.

Dans presque toutes les occurrences d'émerveillement dans le corpus, les personnes qui subissent ce sentiment (experiencer) sont nommées et, dans la majorité des cas, identifiées comme enfants. À côté de cet usage, conforme à la signification traditionnelle, on trouve aussi des emplois sémantiquement plus originaux, comme par exemple le soulignement de l'activité intellectuelle liée à l'émerveillement :

(37) A l'instar d'un chœur, un public est un être vivant éphémère. Son émerveillement n'est pas sommeil, mais éveil, intelligence. (Le Figaro, 2005-02-01) 
49

L'emploi de stupéfaction est moins fréquent et le mot est surtout employé pour désigner des sentiments négatifs. Il se trouve lié avec horreur, chagrin et opposé à amusement et agacement. On trouve de nouveau un lien entre stupéfaction et étonnement. L'experiencer est nommé dans tous les emplois de stupéfaction.

Pour les mots étudiés, qui désignent des mouvements psychiques, on peut constater un emploi synonymique dans certains contextes, mais il y a aussi des emplois spécifiques pour chaque mot qui rendent ses synonymes incompatibles avec le contexte. Surprise est le mot avec l'intension minimale 'mouvement psychique provoqué par quelque chose d'inattendu ou d'extraordinaire'qui peut être considéré comme hyperonyme d'étonnement , d'émerveillement et de stupéfaction. L'étonnement exprime un plus haut degré de force, qui est encore renforcé dans stupéfaction. Ce qui distingue les mots étonnement et émerveillement, est, d'une part, l'accentuation de la cause du mouvement psychique par leur structure morphosémantique et, d'autre part, la différenciation de l'appréciation de ce sentiment. Tandis que pour émerveillement celle-ci est impérativement positive, étonnement est neutre de ce point de vue, mais permet aussi la désignation d'un sentiment négatif. On pourrait ajouter le plus haut degré de surprise exprimé par étonnement.

51 Après avoir étudié toutes les co-occurrences des mots étonnement, émerveillement, surprise et stupéfaction dans GLOSSANET, nous pouvons confirmer les résultats de l'analyse panchronique réalisée à partir de FRANTEXT. Il y a néanmoins quelques modifications à apporter qui dépendent en partie du genre des textes étudiés. Les occurrences de surprise, étonnement, émerveillement et stupéfaction dans GLOSSANET font voir des particularités du développement sémantique de ces mots dans des textes journalistiques. Pour étonnement et stupéfaction, l'emploi pour la désignation de mouvements psychiques évalués comme négatifs est dominant. Parmi les significations de surprise, celle de 'cause du mouvement psychique'est présente dans la majorité des cas. Cette restriction du choix sémantique est liée à une stéréotypisation qui s'exprime aussi dans l'emploi de certains adjectifs (belle). Nous ne pouvons donc pas confirmer la constatation de Blumenthal, Diversy \& Mielebacher (2005 : 70) selon laquelle FRANTEXT, corpus dans lequel les romans sont bien représentés, présenterait davantage de clichés linguistiques qu'un corpus de textes journalistiques. Mais il est évident que la spécificité sémantique d'un terme dans son emploi dépend aussi du genre des textes étudiés.

\section{BIBLIOGRAPHIE}

BLUmenthal, P. (2002) : Profil combinatoire des noms. Synonymie distinctive et analyse contrastive, Zeitschrift für Französische Sprache und Literatur, 112, 115-138.

BLUMENTHAL, P., DIVERSY, S., MIELEBACHER, J. (2005) : Kombinatorische Wortprofile und Profilkontraste. Berechnungsverfahren und Anwendungen, Zeitschrift für romanische Philologie, $121,49-83$

EDMONDS, Ph. et HIRST, G. (2002) : Near-Synonymy and Lexical Choice, Computational Linguistics, 28/1, 105-144. 
GEERARTS, D. (1994) : Synonymy, in R.E. Asher et J.M. Simpson (éds.) : The Encyclopedia of Language and Linguistik, Oxford, New York, Seoul u.a., Pergamon Press, 4455-4456.

WIERZBICKA, A. (1992) : Semantics, Culture and Cognition. Universal Human Concepts in culture-Specific Configurations, Oxford, Oxford University Press.

-, (1996) : Semantics. Primes and universals, Oxford, Oxford University Press.

WitTGENSTEIN, L. (1984) : Tractatus logico-philosophicus. Tagebücher 1914-1916. Philosophische

Untersuchungen, Frankfurt, Suhrkamp.

\section{AUTEUR}

\section{GERDA HAßLER}

Université de Potsdam, Allemagne. 